\title{
Dynamic Decision Making when Risk Perception depends on Past Experience*
}

\author{
Michèle Cohen \\ CES, University Paris 1 \\ Johanna Etner \\ GAINS, University of Maine and CES, University Paris 1 \\ Meglena Jeleva \\ GAINS, University of Maine, and CES, University Paris 1
}

October 2, 2006

\begin{abstract}
The aim of the paper is to propose a preferences representation model under risk where risk perception can be past experience dependent. A first step consists in considering a one period decision problem where individual preferences are no more defined only on decisions but on pairs (decision, past experience). The obtained criterion is used in the construction of a dynamic choice model under risk. The paper ends with an illustrative example concerning insurance demand. It appears that our model allows to explain modifications in the insurance demand behavior over time observed on the insurance markets for catastrophic risk and difficult to justify with standard models.
\end{abstract}

${ }^{*}$ We thank A. Chateauneuf, R. Giraud, J. Y. Jaffray, E. Karni, P. Klibanoff, J. C. Vergnaud and the participants of FUR XII for valuable comments and useful suggestions. 


\section{Introduction}

Decision theory under risk had for a long time focused mainly on the impact of different risk and wealth perceptions on the agents' optimal decisions. In these classical studies, risk perceptions, as well as utility functions, depend only on the considered decision characteristics (pairs probabilities-outcomes) and thus can not be influenced by outside factors. However, some psychological studies (Slovic 2000) point out the fact that risk perception may be strongly influenced by the context in which the individuals are when they take their decisions. Context can take different forms: (i) it can correspond to past experience concerning the same decision problem (relevant in insurance decisions as noticed in Kunreuther (1996) and Brown, Hoyt (2000)) or other events independent on the relevant decision problem as weather or general economic situation (a relation between weather conditions and stock market behaviour have been enlightened by Hirshleifer, Shumway 2003), (ii) it can also correspond to anticipatory feelings about some future states (Caplin, Leahy 2001), (iii) it can be related to the decision outcomes presentation (leading then to the framing effect pointed out by Tversky and Kahneman 1986).

In this paper, we focus on the context created by past experience, corresponding to a sequence of events occurring up to the moment of the decision. This past experience can concern different events : (i) past realizations of the decision-relevant events (as accidents when an insurance decision is considered) or (ii) realization of events, others than the decision relevant ones (such as temperature or health status when a stock demand decision is considered).

The influence of past experience on decisions appears in particular on insurance markets for catastrophic risk. It appears that in California, before the earthquake in 1989, $34 \%$ of individuals consider that insurance against earthquake is useless. After the earthquake, they are only $5 \%$ and $11 \%$ of the non insured individuals subscribed an insurance contract (Kunreuther 1996). These results are confirmed by an empirical study from Brown and Hoyt (2000) that reveals a strong positive correlation between the number of flood insurance contracts subscribed in a year in a given State of the US and losses due to flood in the same State the previous year. A relation between past 
experience and insurance demand appears also in experimental studies when individuals are well informed about the probability of loss realization and about the independence of losses in successive periods (McClelland, Schulze, Coursey 1993, Ganderton et al. 2000, Papon 2004). Their results are less clear-cut: if the existence of a strong correlation between past damages and insurance demand is well established, its sign is less clear. Indeed, two opposite effects can be identified corresponding to availability biais and gambler fallacy in the sense of Tversky and Kahneman (1973). The availability biais corresponds to an overestimation of the probability of an event that recently occurred and implies an increase in insurance demand after a natural disaster, this demand being low after a long period without a catastrophe. The opposite occurs with the gambler's fallacy effect: individuals underestimate the probability of repetition of the event that they just observed and thus buy less insurance after a loss.

When events are independent over time, behaviors that we have just described cannot be explained in the standard expected utility model. Indeed, in this model, past experienced losses lead only to a wealth decrease and not to a probability assesment modification.

Relaxing the axiom of context independence of preferences under uncertainty can allow the rationalization of some decisions considered as inconsistent with respect to the existing criteria because reflecting unstable preferences as for instance the modification of insurance demand against catastrophic risk after the occurrence of a catastroph.

The aim of the paper is to propose a preferences representation model under risk where risk perception can be past experience dependent. A first step consists in considering a one period decision problem where individual preferences are no more defined only on decisions but on pairs (decision, past experience). The obtained criterion is used in the construction of a dynamic choice model under risk.

The underlying model of decision making under risk that is used here is the RDU (Rank dependent Utility), proposed by Kahneman-Tversky (1979), Quiggin (1982) and Yaari (1987). This model has the advantage to allow a non linear treatment of probabilities, in addition to a non linear treatment of outcomes. When one period decisions are considered, we adapt the RDU 
axiomatic system of Chateauneuf (1999) to represent preferences on pairs (decision, past experience).

To better capture the long term impact of past experience on decisions, after the preferences representation on a point of time, we model intertemporal decisions. RDU model can generate intertemporal consistency. To rule out this problem, we use the recursive model of Kreps and Porteus (1978). In the latter model, risk aversion is characterized by a standard utility function and the agents' past experience is summarized by a sequence of monetary payoffs, resulting from the past decisions and the lottery realizations. In the present paper are introduced additional aspects: (i) risk perception is non linear and depends on a probability transformation function; (ii) past experience does not reduce anymore to the only payoffs, but is characterized by a sequence of events, corresponding more generally to individuals' past experience. To achieve this preferences representation, we assume that preferences at a point of time are represented by the "past experience dependent" RDU previously axiomatized and modify the dynamic consistency axiom of KP in order to apply to states and not to payments.

The paper starts with the "past experience dependent" preferences representation at a point of time. We propose an axiomatic foundation for "past experience dependent" rank dependent utility under risk. In the third section, we consider a dynamic choice problem and prove a representation theorem. Section four contains an illustrative example.

\section{Behavior at a point of time}

In this section, we consider a static problem. We propose an axiomatic representation of preferences by a rank dependent expected utility which takes into account the agent's past experience.

\subsection{Notations and definitions}

Decision problem is characterized by a set of risky perspectives in which an agent has to make his choice and by a set of states that characterize the agent's past experience. 
Let $\mathcal{Z}$ denote a set of outcomes. We assume that $\mathcal{Z}$ is a non empty connected compact and metric space and $\mathcal{L}$ is the set of lotteries over $\mathcal{Z}$.

$\mathcal{S}$ is a set of realized states, assumed nonempty, compact, connected separable topological space. An element of $\mathcal{S} \times \mathcal{L}$ will be called a "past experience dependent lottery".

$\succsim$ is a binary relation on $\mathcal{S} \times \mathcal{L}$ which denotes the preference relation of a decision maker. We denote by $\succ$ the strict preference and by $\sim$ the indifference.

Axiom $1 \succsim$ is a weak order on $\mathcal{S} \times \mathcal{L}$.

The preferences representation of $\succsim$ on $\mathcal{S} \times \mathcal{L}$ will be built in two steps. We start with the preference relation on $s \times \mathcal{L}$ and its representation by a Rank Dependant Utility (RDU) model. Then, we give some additional assumptions to achieve a RDU preferences representation on $\mathcal{S} \times \mathcal{L}$.

\subsection{Preferences on $s \times \mathcal{L}$ and RDU}

In this section we consider the restrictions of $\succsim$ to $s \times \mathcal{L}$ that we denote by $\succsim_{s}$. For a given state $s$, we face a standard decision problem under risk.

The RDU representation of the preference relation $\succsim_{s}$ is obtained by the following axioms, proposed in Chateauneuf [1999].

From Axiom 1, it follows directly that $\succsim_{s}$ is a weak order on $s \times \mathcal{L}$.

Axiom 2 (Continuity) For a given $s \in \mathcal{S}$, let $P_{n}=\left(s, L_{n}\right), P=(s, L), Q=$ $\left(s, L^{\prime}\right) \in s \times \mathcal{L}$, with $P_{n}$ weakly converging to $P$, then $\forall n, P_{n} \succsim_{s} Q \Rightarrow P \succsim_{s} Q$ and $\forall n, P_{n} \precsim_{s} Q \Rightarrow P \precsim_{s} Q$.

For a given $s$, it is possible to completely order the space $\mathcal{Z}$. The definition of the first order stochastic dominance (SD1) becomes $L S D 1 L^{\prime}$ if and only if $P_{L}\left(z \in \mathcal{Z},(s, z) \succ_{s}(s, x)\right) \geq P_{L^{\prime}}\left(z \in \mathcal{Z}, \quad(s, z) \succ_{s}(s, x)\right) \forall x \in \mathcal{Z}$.

The next axiom guarantees that $\succsim_{s}$ preserves first order stochastic dominance.

Axiom 3 For any $L, L^{\prime} \in \mathcal{L}$ such that $L S D 1 L^{\prime},(s, L) \succsim_{s}\left(s, L^{\prime}\right)$. 
Axiom 4 (Comonotonic sure-thing principle) For any $s \in \mathcal{S}$, let lotteries $P=\left(\left(s, z^{i}\right) p_{i}\right), Q=\left(\left(s, y^{i}\right) p_{i}\right)$ be such $\left(s, z^{i_{0}}\right) \sim_{s}\left(s, y^{i_{0}}\right)$, then $P \succsim_{s} Q$ implies $P^{\prime} \succsim_{s} Q^{\prime}$, for lotteries $P^{\prime}, Q^{\prime}$ obtained from lotteries $P$ and $Q$ by merely replacing the $i_{0}^{\text {th }}$ common pair $\left(s, z^{i_{0}}\right)$, by a common pair $\left(s, x^{i_{0}}\right)$ again in $t_{0}^{\text {th }}$ rank both in $P^{\prime}$ and $Q^{\prime}$.

Axiom 5 (Comonotonic mixture independance axiom) For any $s \in$ $\mathcal{S}$, and for any lotteries $P=\left(\left(s, z^{i}\right) p_{i}\right)$ and $\left.Q=\left(\left(s, y^{i}\right) q_{i}\right)\right)$,

For any $p \in[0,1]$, for any $a, b, c, d \in \mathcal{Z}$

- $P_{1}=(1-p)\left(s, z^{\min }\right)+p(s, a) \sim_{s} Q_{1}=(1-p)\left(s, y^{\min }\right)+p(s, b)$

and $P_{2}=(1-p)\left(s, z^{\min }\right)+p(s, c) \sim_{s} Q_{2}=(1-p)\left(s, y^{\min }\right)+p\left(s^{\prime}, d\right)$ imply $\forall \alpha \in[0,1], \alpha P_{1}+(1-\alpha) P_{2} \sim_{s} \alpha Q_{1}+(1-\alpha) Q_{2}$

- $P_{1}=(1-p)\left(s, z^{\max }\right)+p(s, a) \sim_{s} Q_{1}=(1-p)\left(s, y^{\max }\right)+p\left(s^{\prime}, b\right)$

and $P_{2}=(1-p)\left(s, z^{\max }\right)+p(s, c) \sim_{s} Q_{2}=(1-p)\left(s, y^{\max }\right)+p\left(s^{\prime}, d\right)$ imply $\forall \alpha \in[0,1], \alpha P_{1}+(1-\alpha) P_{2} \sim_{s} \alpha Q_{1}+(1-\alpha) Q_{2}$

Theorem 1 Let the preference relation $\succsim_{s}$ on $s \times \mathcal{L}$ satisfy axioms $1-5$, then there exist an increasing function $\varphi_{s}:[0,1] \rightarrow[0,1]$, with $\varphi_{s}(0)=0, \varphi_{s}(1)=$ 1 and $a$ utility function, $v_{s}: \mathcal{Z} \rightarrow \mathbf{R}$, which is increasing, continuous, and unique up to an affine transformation such that:

$\forall L, L^{\prime} \in \mathcal{L},(s, L) \succsim_{s}\left(s, L^{\prime}\right)$ iff $V_{s}(L) \geq V_{s}\left(L^{\prime}\right)$ with

$$
V_{s}(L)=\sum_{i=1}^{n}\left(\varphi_{s}\left(\sum_{j=i}^{n} p_{j}\right)-\varphi_{s}\left(\sum_{j=i+1}^{n} p_{j}\right)\right) \times v_{s}\left(z^{i}\right)
$$

Proof. Chateauneuf [1999]

We now consider the general preferences comparing lotteries in different contexts $(\mathcal{S} \times \mathcal{L})$. Let us notice that the preferences on $\mathcal{S} \times \mathcal{L}$ induce a preference on $\mathcal{S} \times \mathcal{Z}$ but in no case a preference on $\mathcal{S}$ alone. 
At this stage, the payoffs evaluation depends not only on $z$ but also on $s$. The objective of this paper is to emphasize the link between risk perception and individual context. In order to isolate this feature, we assume that only perceptions depend on $s$. This assumption needs more discussion, mainly with respect to the state-dependant model of Karni (1985). The main feature of Karni's model is that the evaluation of a given amount of money may strongly depend on the state in which the individual is when receiving this amount. Here, the states we consider are not of the same type: they are already realized (past) states, and not future ones. It seems then more realistic to assume that they are more likely to influence risk perception than future monetary evaluations.

The following axiom guarantees that the payoffs evaluations do not depend on past experience.

Axiom 6 For any $s, s^{\prime} \in \mathcal{S}$ and any $z \in \mathcal{Z},(s, z) \sim\left(s^{\prime}, z\right)$.

Let us notice that axioms 3 and 6 induce the existence of a preference relation on $\mathcal{Z}$ independent on $\mathcal{S}$. To simplify notations, we can then write $z \geq z^{\prime}$ instead of $(s, z) \succeq\left(s, z^{\prime}\right)$ for all $s \in \mathcal{S}$.

The following preferences representation theorem can then be formulated.

Theorem 2 Under Axioms 1 - 6, the weak order $\succsim$ on $\mathcal{S} \times \mathcal{L}$ is representable by a function $V: \mathcal{S} \times \mathcal{L} \rightarrow \mathbf{R}$. For any $s, s^{\prime} \in \mathcal{S}$ and any $L, L^{\prime} \in \mathcal{L}$, $(s, L) \succsim\left(s^{\prime}, L^{\prime}\right) \Leftrightarrow V(s, L) \geq V\left(s^{\prime}, L^{\prime}\right)$

where $V(s, L)=\sum_{i=1}^{n}\left(\varphi_{s}\left(\sum_{j=i}^{n} p_{j}\right)-\varphi_{s}\left(\sum_{j=i+1}^{n} p_{j}\right)\right) v\left(z^{i}\right)$.

Proof. The generalization of the preferences representation of the restrictions $\succsim_{s}$ on $\succsim$ is allowed by the unicity of the probability transformation function $\varphi_{s}(p)$ and the independence of the utility function on $s$.

We can see that a decision maker with $\varphi_{s}(p)<p$ systematically underestimates the probabilities of the favorable events and then is called pessimist under risk. Moreover, we obtain the following result.

Corollary 1 Let $s, s^{\prime} \in \mathcal{S} . \varphi(s, p) \geq \varphi\left(s^{\prime}, p\right)$ for any $p \in[0,1]$ if and only $V(s, L) \geq V\left(s^{\prime}, L\right)$ for any $L \in \mathcal{L}$. 
Proof. Let $L=\left(z_{1}, p_{1} ; z_{2}, p_{2} ; \ldots ; z_{n}, p_{n}\right)$ with $z_{1} \leq \ldots \leq z_{n}$ Note that this axiom implies that for any $z, z^{\prime} \in \mathcal{Z}$ such that $z \geq z^{\prime},(s, z) \succsim_{s}\left(s, z^{\prime}\right)$.

(i) $\Rightarrow: V(s, L)-V\left(s^{\prime}, L\right)=\sum_{i=2}^{n}\left(\varphi_{s}\left(\sum_{j=i}^{n} p_{j}\right)-\varphi_{s^{\prime}}\left(\sum_{j=i}^{n} p_{j}\right)\right)\left(v\left(z^{i}\right)-v\left(z^{i-1}\right)\right) \geq$ 0 if $\varphi(s, p) \geq \varphi\left(s^{\prime}, p\right)$ for any $p \in[0,1]$;

(ii) $\Leftarrow$ : if there exists $p_{0}$ such that $\varphi\left(s, p_{0}\right)<\varphi\left(s^{\prime}, p_{0}\right)$ then for $L_{0}=$ $\left(z_{1}, 1-p_{0} ; z_{2}, p_{0}\right), V\left(s, L_{0}\right)<V\left(s^{\prime}, L_{0}\right)$.

This result implies that if there exists a realized state that induces pessimistic risk perception, then an individual will dislike any decision in this context, with respect to a state where his risk perception is less pessimistic.

\section{Dynamic choice}

In this section, we consider a dynamic choice problem under risk where risk perception and utility of the outcomes may depend on agents' past experience. Preferences at a point of time are represented as in the previous section of the paper, by a past experience dependent RDU. It is now well known (see for instance Machina 1989) that preferences representations models that do not verify the independence axiom can not verify at the same time dynamic consistency, consequentialism and reduction of compound lotteries. To preserve dynamic consistency, as in Epstein, Wang (1994) and Epstein, Schneider (2003), a recursive model is adopted here. More precisely, we modify the Kreps and Porteus (1978) model in order to introduce both risk perception and past experience dependence.

\subsection{Some notations and definitions}

We consider a discrete and finite sequence of times $t=1, \ldots, T . Z_{t}$ is the set of possible payoffs at time $t$. To simplify, we assume that $Z_{t}=Z$ which is a compact interval of $R$ for any $t$ from 1 to $T$. A payoff realized at time $t$ is denoted by $z_{t}$. Decision maker past experience at time $t$ is characterized by a sequence of events, relevant for the considered decision and denoted by $s_{t}$. More precisely, $s_{t}=\left(e_{0}, e_{1}, \ldots, e_{t}\right)$ where $e_{\tau}$ is the event that occurred at time $\tau$ with $e_{\tau} \in \mathcal{E}_{\tau}$, the set of all possible events at time $\tau$ and $e_{0} \in \mathcal{E}_{0}$ the 
set of all possible past experiences. $S_{t}$ is then the set of possible histories up to time $t$ verifying the recursive relation: $S_{0}=\mathcal{E}_{0}$ and $S_{t}=S_{t-1} \times \mathcal{E}_{t}$. We denote by $\mathcal{M}\left(\mathcal{E}_{t}\right)$ the set of distributions on $\mathcal{E}_{t}$.

At period $T, \mathcal{L}_{T}$ is the set of distributions on $Z_{T}$ endowed with the Prohorov metric. $X_{T}$, the set of risky perspectives in which agent has to make his choice, is assumed to be the set of closed non empty subsets of $\mathcal{L}_{T}$, endowed with the Hausdorff metric.

By recurrence, we define, $\mathcal{L}_{t}$, the set of probability distributions on

$\mathcal{C}_{t}=Z_{t} \times X_{t+1} \times \mathcal{M}\left(\mathcal{E}_{t+1}\right)$ with $X_{t+1}$ the set of closed non empty subsets of $\mathcal{L}_{t+1}$.

At each period, the nature chooses a probability distribution on $\mathcal{E}_{t+1}$. The agent cannot influence this distribution. Given this distribution, the agent has to choose a lottery in the set $\mathcal{L}_{t}$. The assumption of compound lotteries reduction is made between distributions on wealth and events for a fixed period. However, this assumption is relaxed between two consecutive periods.

For each period $t, \succsim_{t}$ denotes a binary relation on $s_{t} \times \mathcal{L}_{t}$ for a given $s_{t}$. We denote $\succ_{t}$ the strict preference and $\sim_{t}$ the indifference.

Axiom 7 (1bis) $\succsim_{t}$ is a complete order on $s_{t} \times \mathcal{L}_{t}$.

We assume that $\succsim_{t}$ verifies axioms $2-5$ on $s_{t} \times \mathcal{L}_{t}$.

Under these previous axioms, we can represent preferences at a point of time in the similar way to theorem 1 :

Lemma 1 For any $s_{t}$ in $S_{t}$, axioms 1bis, 2-5 are necessary and sufficient for there to exist, for each $t$, a bounded continuous function $v_{t}: s_{t} \times Z_{t} \times$ $X_{t+1} \times \mathcal{E}_{t+1} \longrightarrow R$ and a continuous function $\varphi_{t}: s_{t} \times[0,1] \longrightarrow[0,1]$ such that for $L_{t}, L_{t}^{\prime} \in \mathcal{L}_{t},\left(s_{t}, L_{t}\right) \succsim_{t}\left(s_{t}, L_{t}^{\prime}\right)$ if and only if $V_{t}\left(s_{t}, L_{t}\right) \geq V_{t}\left(s_{t}, L_{t}^{\prime}\right)$ with

$$
V_{t}\left(s_{t}, L_{t}\right)=\sum_{i=1}^{n}\left(\varphi_{t}\left(s_{t}, \sum_{j=i}^{n} p_{j}\right)-\varphi_{t}\left(s_{t}, \sum_{j=i+1}^{n} p_{j}\right)\right) \times v_{t}\left(s_{t}, z_{t}, x_{t+1}, e_{t+1}\right) \equiv
$$
$R D v_{t}\left(s_{t}, L_{t}\right)$.

The proof comes immediately from the previous section. Let us notice that utility function $v_{t}$ depend on past experience $s_{t}$ whereas in a static 

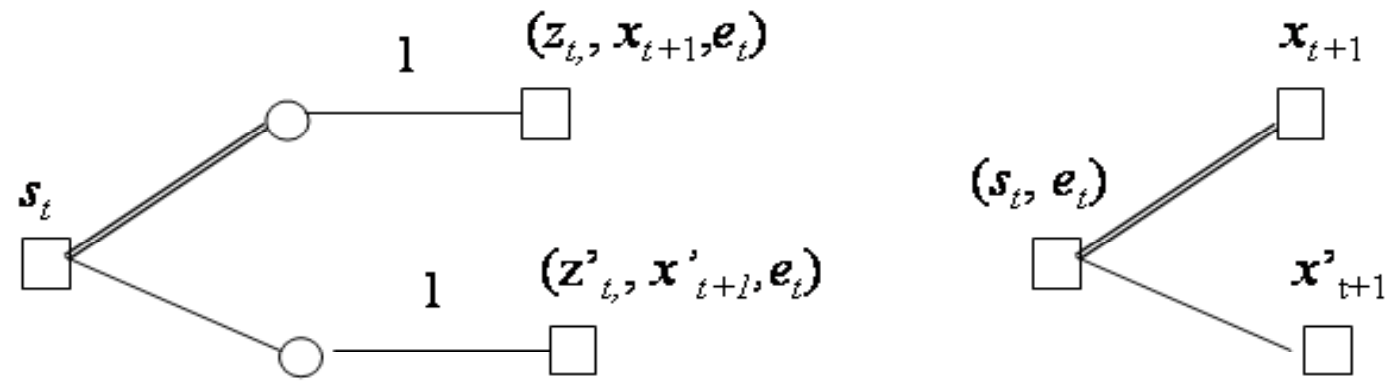

Figure 1:

problem, $v$ did not depend on state $s$. This comes from the fact that in a dynamic choice, future perspective depend on past experience. We will precise this point in the next section.

\subsection{Temporal consistency and the representation the- orem}

We adapt the KP temporal consistency axiom to our context in the following manner.

Axiom 8 (Temporal consistency) We consider the degenerate distributions, $\Delta_{e_{t}}$ in $\mathcal{M}\left(\mathcal{E}_{t}\right)$ and for a given distribution $\delta_{e_{t+1}}$ in $\Delta_{e_{t+1}}$, the degenerate distributions on $Z_{t} \times X_{t+1} \times \delta_{e_{t+1}}$. Then, for all $t, s_{t} \in S_{t}, e_{t+1} \in \mathcal{E}_{t+1}, z_{t} \in Z_{t}$, $x_{t+1}, x_{t+1}^{\prime} \in X_{t+1}$,

$\left(s_{t}, z_{t}, x_{t+1}, e_{t+1}\right) \succsim_{t}\left(s_{t}, z_{t}, x_{t+1}^{\prime}, e_{t+1}\right) \quad$ iff $\left(s_{t+1}, x_{t+1}\right) \succsim_{t+1} \quad\left(s_{t+1}, x_{t+1}^{\prime}\right)$ with $s_{t+1}=\left(s_{t}, e_{t+1}\right)$ at period $t+1$.

Let us consider the two following lotteries:

The temporal consistency axiom sets that if the degenerate lottery $\left(z_{t}, x_{t}, e_{t}\right)$ is preferred to the degenerate lottery $\left(z_{t}^{\prime}, x_{t}^{\prime}, e_{t}\right)$ for a decision maker with past 
experience $s_{t}$, then if at time $t, e_{t}$ is realized, the decision maker will choose $x_{t}$ between $x_{t}$ and $x_{t}^{\prime}$. In the same way, if at time $t+1$, he chooses $x_{t}$, then he cannot at time $t$, strictly prefer $\left(z_{t}^{\prime}, x_{t}^{\prime}, e_{t}\right)$ to $\left(z_{t}, x_{t}, e_{t}\right)$.

Lemma 2 Axioms 1bis, 2-5 and 8 are necessary and sufficient for there to exist functions $v_{t}$ as in previous lemma and, for fixed $\left\{v_{t}\right\}$, unique functions $u_{t}:\left\{\left(s_{t}, z_{t}, \gamma\right) \in S_{t} \times Z_{t} \times R: \gamma=R D v_{t+1}\left(s_{t+1}, L_{t+1}\right)\right\} \rightarrow R$

which are strictly increasing in their third argument and which satisfy $v_{t}\left(s_{t}, z_{t}, x_{t+1}, e_{t+1}\right)=u_{t}\left(s_{t}, z_{t}, L_{t+1} \max R D v_{t+1}\left(s_{t+1}, L_{t+1}\right)\right)$ for all $s_{t} \in$ $S_{t}, e_{t+1} \in \mathcal{E}_{t+1}, z_{t} \in Z_{t}, x_{t+1} \in X_{t+1}$.

Proof. Axioms 1bis-5 and 8 are hold, lemma 3 fix $V_{t}\left(s_{t}, L_{t}\right)$. Then, $V_{t+1}\left(\left(s_{t}, e_{t+1}\right), L_{t+1}\right)=V_{t+1}\left(\left(s_{t}, e_{t+1}\right), L_{t+1}^{\prime}\right) \Longrightarrow V_{t}\left(s_{t}, L_{t+1}\right)=V_{t}\left(s_{t}, L_{t+1}^{\prime}\right)$ for a given $e_{t+1}$ (axiom TC). Consequently, $u_{t}$ is strictly increasing in its third argument.

(ii) If $V_{t}$ and $u_{t}$ are given with $u_{t}$ is strictly increasing in its third argument, $V_{t}$ verifies lemma 3 . Then axioms 1bis-6 hold. $u_{t}$ is increasing in its third argument then $V_{t}\left(s_{t}, L\right) \geq V_{t}\left(s_{t}, L^{\prime}\right) \Longleftrightarrow u_{t}\left(s_{t}, z_{t}, V_{t+1}\left(s_{t+1}, L\right)\right) \geq$ $u_{t}\left(s_{t}, z_{t}, V_{t+1}\left(s_{t+1}, L^{\prime}\right)\right)$

$\Longleftrightarrow V_{t+1}\left(\left(s_{t}, e_{t+1}\right), L\right) \geq V_{t+1}\left(\left(s_{t}, e_{t+1}\right), L^{\prime}\right)$. Then axiom 8 holds.

As we can see, in the dynamic problem, utility functions $v_{t}$ depend on past experience $s_{t}$. Indeed, at time $T$, utility function does not depend on past experience. But, at time $T-1$, the certainty equivalent of lottery, given by $u_{T-1}$, depends on past experience $s_{T-1}$. Consequently, $v_{T-1}$ directly depends on past experience. Recursively, at each period, certainty equivalent depends on past experience and then utility function too.

Theorem 3 Axioms 1bis, 2-5 and 8 are necessary and sufficient for there to exist a continuous function $v: S_{T} \times Z_{T} \rightarrow R$ and, for $t=0, \ldots, T-1$, continuous functions $u_{t}: S_{t} \times Z_{t} \times R \rightarrow R$, strictly increasing in their third argument, so that, $v_{T}\left(s_{T}, z_{T}\right)=v\left(z_{T}\right)$ and, recursively

$$
v_{t}\left(s_{t}, z_{t}, x_{t+1}, e_{t+1}\right)=L_{t+1} \max u_{t}\left(s_{t}, z_{t}, R D v_{t+1}\left(s_{t+1}, L_{t+1}\right)\right) \text {, }
$$


then, for all $s_{t} \in S_{t}, L_{t}, L_{t}^{\prime} \in \mathcal{L}_{t},\left(s_{t}, L_{t}\right) \succsim_{t}\left(s_{t}, L_{t}^{\prime}\right)$ iff $R D v_{t}\left(s_{t}, L_{t}\right) \geq$ $R D v_{t}\left(s_{t}, L_{t}^{\prime}\right)$

with $R D v_{t}\left(s_{t}, L_{t}\right)=\sum_{i=1}^{n}\left(\varphi_{t}\left(s_{t}, \sum_{j=i}^{n} p_{j}\right)-\varphi_{t}\left(s_{t}, \sum_{j=i+1}^{n} p_{j}\right)\right) \times v_{t}\left(s_{t}, l_{t}\right)$.

Proof. We adapt the demonstration of the theorem in Kreps and Porteus.

Notice that the representation needs $v$, the functions $u_{t}$ and the functions $\varphi_{t}$ to implicitly define functions $v_{t}$. As in Kreps and Porteus, it introduces the concept of timing of resolution of uncertainty. This representation can explain some dynamic behaviors not explain by the standard Expected Utility model. We propose in the next section an illustration.

\section{An insurance demand illustration}

In this section, we study the implications of the previous model for multiperiod demand decisions on the insurance market. It appears that introducing a relation between realized damages and risk perception gives an explanation for the observed insurance demand against catastrophic risk.

We study the optimal insurance demand strategy of an individual for 3 periods of time (years). The individual faces a risk of loss of amount $L$ at each period. There exist a perfectly competitive insurance market proposing insurance contracts at a fair premium corresponding to the estimated expected loss. Insurance contracts are subscribed for one period. Consequently, the individual has to choose an amount of coverage at each period. We assume that for one period the estimated probability of incurring a loss is $p$ and that losses in successive periods are independent: $\mathrm{P}$ (loss at period $t / \operatorname{loss}$ in period $t-1)=p$.

We can represent the decision tree as following:

At each period, the insurance contract $C_{t}$ is characterized by a pair (indemnity $I_{t}$, premium $\Pi_{t}$ ). Only proportional coverage is considered: $I_{t}=\alpha_{t} L$ with $\alpha_{t} \in[0,1]$. The premium for a coverage rate $\alpha_{t}$ is $\Pi_{t}=\alpha_{t} p L$. At period $t=0$, the agent receives a certain wealth, $z_{0}$, and he is in the state $s_{0}$. Past experience is resumed by the sequences of events \{damage, no damage\}. We 


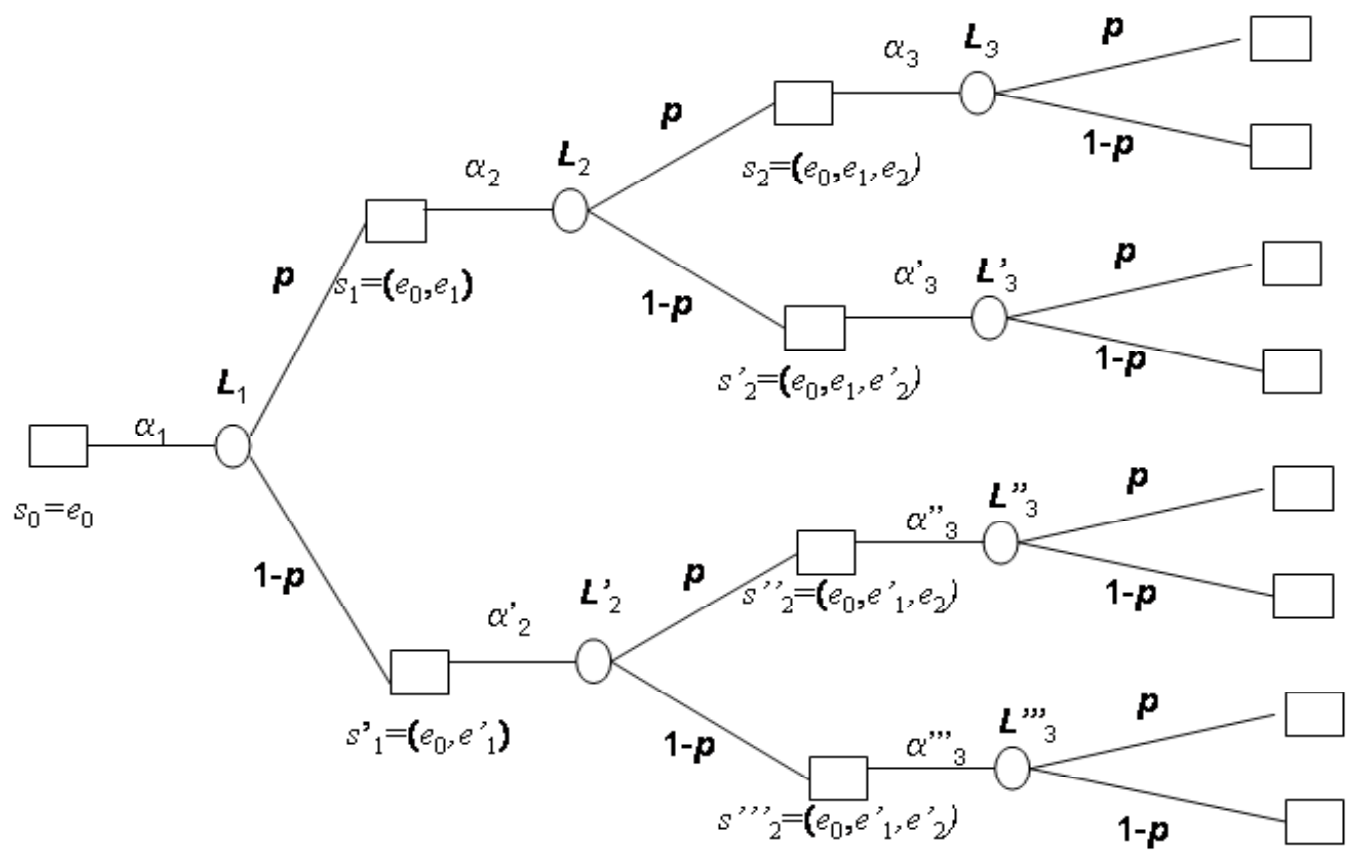

Figure 2: 
denote by $e_{t}$ the event "damage at period $t$ " and by $e_{t}^{\prime}$ the event "no damage at period $t$ ". At each period, individual has to choose $\alpha_{t}$.

At a point of time, the probability transformation function is assumed to be the following:

$$
\varphi_{t}\left(p, s_{t}\right)=p^{\sum^{t} e_{\tau}}
$$

with $e_{0}=\frac{1}{2}, e_{t}=\frac{1}{2}$ and $e_{t}^{\prime}=0$ for $t=1,2$.

In this case, the individual is optimistic at period 0 and modifies his risk perception with respect to damages, occurring or not: the occurrence of a damage modifies his risk perception and he becomes less optimistic, if no damages occurs, his risk perception does not change.

To better isolate the risk perception arguments, we assume that preferences at the final period are represented by a linear utility function under certainty.

Moreover, for simplicity, we suppose that the individual is neutral toward the time of resolution of uncertainty (in the sense of Kreps and Porteus), so that $u_{t}\left(s_{t}, z_{t}, R D v_{t+1}\left(s_{t+1}, L_{t+1}\right)\right)=R D v_{t+1}\left(s_{t+1}, L_{t+1}\right)$.

The dynamic choice problem solves in several steps. Note that, due to the linear utility assumption, only corner solutions will prevail.

(i) For each terminal node, we have to compute $v\left(s_{3}, z_{3}\right)=z_{3}$, with $z_{3}$ the wealth at the final period.

(ii) For each final decision node, we have to evaluate the individual utility and maximize it.

For example, at node $L_{3}$, for a coverage rate $\alpha_{3}$, the utility writes:

$$
\begin{gathered}
V_{3}\left(s_{3}, L_{3}\right)=R D\left(s_{3}, L_{3}\right)=v\left(s_{3}, \underline{z_{3}}\right)+ \\
{\left[v\left(s_{3}, \bar{z}_{3}\right)-v\left(s_{3}, \underline{z_{3}}\right)\right] \times \varphi_{3}\left(1-p, s_{3}\right)} \\
\text { with } \underline{z_{3}}=z_{2}-\Pi_{3}-L+I_{3}=z_{2}-L+\alpha_{3} L(1-p), \bar{z}_{3}=z_{2}-\Pi_{3}= \\
z_{2}-\alpha_{3} L p, \text { and } \varphi_{3}\left(1-p, s_{3}\right)=(1-p)^{e_{0}+e_{1}+e_{2}}=(1-p)^{\frac{3}{2}} .
\end{gathered}
$$


Then,

$$
\begin{aligned}
V_{3}\left(s_{3}, L_{3}\right) & =z_{2}-L+\alpha_{3} L(1-p)+L\left[1-\alpha_{3}\right] \times(1-p)^{\frac{3}{2}} \\
& =\alpha_{3} L(1-p)\left[1-(1-p)^{\frac{1}{2}}\right]+z_{2}-L+L(1-p)^{\frac{3}{2}}
\end{aligned}
$$

As utility is an increasing function of the coverage rate, the optimal coverage is the full coverage and the value of utility becomes

$$
V_{3}\left(s_{3}, L_{3}^{*}\right)=z_{2}-p L
$$

In the same way, we obtain that $V_{3}\left(s_{3}^{\prime}, L_{3}^{\prime *}\right)=z_{2}-p L$ for any $\alpha_{3}^{\prime} \in$ $[0,1], V_{3}\left(s_{3}^{\prime \prime}, L_{3}^{\prime \prime *}\right)=z_{2}-p L$ for any $\alpha_{3}^{\prime \prime} \in[0,1]$ and $V_{3}\left(s_{3}^{\prime \prime \prime}, L_{3}^{\prime \prime \prime *}\right)=z_{2}-$ $L\left(1-(1-p)^{1 / 2}\right)$ for $\alpha_{3}^{\prime \prime \prime *}=0$.

(iii) At period 2, we have four nodes which values are:

$u_{2}\left(s_{2}, \underline{z}_{2}, R D v_{3}\right)=\underline{z}_{2}-p L$ with $\underline{z}_{2}=z_{1}-\Pi_{2}-L+I_{2}$,

$u_{2}\left(s_{2}, \bar{z}_{2}, R D v_{3}\right)=\bar{z}_{2}-p L$ with $\bar{z}_{2}=z_{1}-\Pi_{2}$,

$u_{2}\left(s_{2}, \underline{z}_{2}^{\prime}, R D v_{3}\right)=\underline{z}_{2}^{\prime}-p L$ with $\underline{z}_{2}^{\prime}=z_{1}-\Pi_{2}^{\prime}-L+I_{2}^{\prime}$ and

$u_{2}\left(s_{2}, \bar{z}_{2}^{\prime}, R D v_{3}\right)=\bar{z}_{2}^{\prime}-L\left(1-(1-p)^{1 / 2}\right)$ with $\bar{z}_{2}^{\prime}=z_{1}-\Pi_{2}^{\prime}$.

(iv) We repeat step (ii). Then, at node $L_{2}$, for a coverage rate $\alpha_{2}$, the utility writes:

$V_{2}\left(s_{2}, L_{2}\right)=R D v_{2}\left(s_{2}, L_{2}\right)=v_{2}\left(s_{2}, \underline{z_{2}}\right)+\left[v_{2}\left(s_{2}, \bar{z}_{2}\right)-v_{2}\left(s_{2}, \underline{z_{2}}\right)\right] \times \varphi_{2}\left(1-p, s_{2}\right)$

with $v_{2}\left(s_{2}, \underline{z}_{2}\right)=u_{2}\left(s_{2}, \underline{z}_{2}, R D v_{3}\right)=\underline{z}_{2}-p L=z_{1}-L(1+p)+$ $\alpha_{2} L(1-p), v_{2}\left(s_{2}, \bar{z}_{2}\right)=u_{2}\left(s_{2}, \bar{z}_{2}, R D v_{3}\right)=\bar{z}_{2}-p L=z_{1}-p L-\alpha_{2} p L$ and $\varphi_{2}\left(1-p, s_{2}\right)=(1-p)^{e_{0}+e_{1}}=1-p$.

Then,

$$
V_{2}\left(s_{2}, L_{2}\right)=z_{1}-2 L p
$$

for any $\alpha_{2} \in[0,1]$. 
At node $L_{2}^{\prime}$, we have to pay attention to the value of $v_{2}\left(s_{2}^{\prime}, \underline{z}_{2}^{\prime}\right)=$ $u_{2}\left(s_{2}^{\prime}, \underline{z}_{2}^{\prime}, R D v_{3}\right)$ and $v_{2}\left(s_{2}^{\prime}, \bar{z}_{2}^{\prime}\right)=u_{2}\left(s_{2}^{\prime}, \bar{z}_{2}^{\prime}, R D v_{3}\right)$ since, in the RDU framework, we have to rank utility.

In our example, $v_{2}\left(s_{2}^{\prime}, \underline{z}_{2}^{\prime}\right)<v_{2}\left(s_{2}^{\prime}, \bar{z}_{2}^{\prime}\right)$. Thus, we obtain that

$$
\begin{aligned}
& V_{2}\left(s_{2}^{\prime}, L_{2}^{\prime}\right)=R D v_{2}\left(s_{2}^{\prime}, L_{2}^{\prime}\right)=v_{2}\left(s_{2}^{\prime}, \underline{z_{2}^{\prime}}\right)+\left[v_{2}\left(s_{2}^{\prime}, \bar{z}_{2}^{\prime}\right)-v_{2}\left(s_{2}^{\prime}, \underline{z_{2}^{\prime}}\right)\right] \times \varphi_{2}\left(1-p, s_{2}^{\prime}\right) \\
& \text { with } v_{2}\left(s_{2}^{\prime}, \underline{z_{2}^{\prime}}\right)=\underline{z}_{2}^{\prime}-p L=z_{1}-L(1+p)+\alpha_{2}^{\prime} L(1-p), v_{2}\left(s_{2}^{\prime}, \bar{z}_{2}^{\prime}\right)= \\
& \bar{z}_{2}^{\prime}-L\left(1-(1-p)^{1 / 2}\right)=z_{1}-L\left(1-(1-p)^{1 / 2}\right)-\alpha_{2}^{\prime} p L \text { and } \varphi_{2}\left(1-p, s_{2}^{\prime}\right)= \\
& (1-p)^{e_{0}+e_{1}^{\prime}}=(1-p)^{1 / 2} .
\end{aligned}
$$

Then,

$$
V_{2}\left(s_{2}^{\prime}, L_{2}^{\prime}\right)=z_{1}+L p\left[(1-p)^{1 / 2}-2\right]+\alpha_{2}^{\prime} L(1-p)^{1 / 2}\left[(1-p)^{1 / 2}-1\right]
$$

As utility is a decreasing function of the coverage rate, the optimal coverage is null and the value of utility becomes

$$
V_{2}\left(s_{2}^{\prime}, L_{2}^{\prime *}\right)=z_{1}+L p\left[(1-p)^{1 / 2}-2\right]
$$

(v) At period 1, we have two nodes which values are:

$u_{1}\left(s_{1}, \underline{z}_{1}, R D v_{2}\right)=\underline{z}_{1}-2 p L$ with $\underline{z}_{1}=z_{0}-\Pi_{1}-L+I_{1}$ and $u_{1}\left(s_{1}, \bar{z}_{1}, R D v_{2}\right)=\bar{z}_{1}+L p\left[(1-p)^{1 / 2}-2\right]$ with $\bar{z}_{1}=z_{0}-\Pi_{1}$.

Then, at node $L_{1}$, for a coverage rate $\alpha_{1}$, the utility writes:

$$
\begin{aligned}
& V_{1}\left(s_{1}, L_{1}\right)=R D v_{1}\left(s_{1}, L_{1}\right)=v_{1}\left(s_{1}, \underline{z_{1}}\right)+\left[v_{1}\left(s_{1}, \bar{z}_{1}\right)-v_{1}\left(s_{1}, \underline{z_{1}}\right)\right] \times \varphi_{1}\left(1-p, s_{1}\right) \\
& \quad \text { with } v_{1}\left(s_{1}, \underline{z}_{1}\right)=\underline{z}_{1}-2 p L=z_{0}-\Pi_{1}-L+I_{1}-2 p L=\alpha_{1} L(1-p)+z_{0}- \\
& L(1+2 p), v_{1}\left(s_{1}, \bar{z}_{1}\right)=\bar{z}_{1}+L p\left[(1-p)^{1 / 2}-2\right]=z_{0}-\alpha_{1} p L+L p\left[(1-p)^{1 / 2}-2\right] \\
& \text { and } \varphi_{1}\left(1-p, s_{1}\right)=(1-p)^{e_{0}}=(1-p)^{1 / 2} .
\end{aligned}
$$

Then,

$V_{1}\left(s_{1}, L_{1}\right)=\alpha_{1} L(1-p)^{1 / 2}\left[(1-p)^{1 / 2}-1\right]+z_{0}-L(1+2 p)+L\left[p(1-p)^{1 / 2}+1\right](1-p)^{1 / 2}$

As utility is a decreasing function of the coverage rate, the optimal coverage is zero and the value of utility becomes

$$
V_{1}\left(s_{1}, L_{1}^{*}\right)=z_{0}-L(1+2 p)+L\left[p(1-p)^{1 / 2}+1\right](1-p)^{1 / 2}
$$

To summarize, the results are the following: 
- $\alpha_{1}=0$;

- $\alpha_{2}=0$ if no loss at period 1 ;

$\alpha_{2} \in[0,1]$ if loss at period 1 ;

- $\alpha_{3}=0$ if no loss at periods 1 and 2 ;

$\alpha_{3}=1$ if loss at periods 1 and 2 ;

$\alpha_{3} \in[0,1]$ else.

In this illustration, the individual chooses not to buy insurance contract in the first period. In the second period, he choose not to be covered only if he had not damage. Finally, in the third period, if he had never had an accident, he chooses not buying insurance contract, if he had two consecutive accidents, he decides to buy a full coverage and in the intermediate cases, he is indifferent.

This example underlines the fact that what is important for the decision maker is not only the event occurring in the period directly preceding the moment of the decision, but the all sequence of events, that is all the past experience.

Let us now compare the predictions of our model with those of some standard modelizations.

- the particular case when $\varphi_{t}\left(p, s_{t}\right)=p$ : this corresponds to the standard version of a recursive expected utility model.

The results are then the following: $\alpha_{i} \in[0,1]$ for any $i=1,2,3$. The individual is indifferent between different amounts of coverage and this, at any period and for any experienced damage.

- we consider here an alternative non consequentialist way to model dynamic choices: the resolute choice model proposed by McClennen, 1990. In this model, all the strategies are evaluated at the root node and compared according to the root node preferences. We consider 2 cases: the case when one-shot preferences are EU and the case when one-shot preferences are RDU. The results are then the following: 
(i) for EU preferences, $\alpha_{i} \in[0,1]$ for any $i=1,2,3$. The individual is indifferent between different amounts of coverage and this, at any period and for any experienced damage.

(ii) for RDU preferences, $\alpha_{i}=0$ or 1 for any $i$.

- for $\varphi(p)<p$, complete coverage at any period and for any experienced loss is preferred to a strategy consisting in buying insurance only after experiencing a loss;

- for $\varphi(p)>p$, no coverage at any period and for any experienced loss is preferred to a strategy consisting in buying insurance only after experiencing a loss.

\section{Concluding remarks}

The insurance demand example shows that our model allows to explain the modifications in the insurance demand behaviour over time observed for catastrophic risk and given in the introduction. It well appears that past experience have a cumulative effect on decisions: an individual can maintain constant its insurance demand after one occurrence of the loss and modify it only after two, or more consecutive loss events. In this example, we assumed that at any period observing a loss renders the individual more pessimistic. This explains a behaviour in accordance with the availability bias. The gambler's fallacy attitude is explained if the individual becomes more and more optimistic after experiencing losses.

The comparison with other modelizations shows that neither the recursive model alone, nor the RDU model alone can justify all the observed pattern of behaviour.

The insurance example corresponds to the particular case when past experience (context) is composed by the decision-relevant events. Considering different events, that do not directly influence the outcomes (as weather condition in investment decisions) will make even easier to underline the new insights of the present model because of the complete absence of these events in the preferences representations of the standard models. 


\section{References}

[1] Browne M., Hoyt R. (2000), "The demand for flood insurance: empirical evidence", Journal of risk and uncertainty, 20, 291-306.

[2] Caplin A., Leahy J. (2001), "Psychological expected utility theory and anticipatory feeling", The Quarterly Journal of Economics, 55-79.

[3] Chateauneuf A. (1999), "Comonotonicity axioms and rank-dependent expected utility theory for arbitrary consequences", Journal of Mathematical Economics,21-45.

[4] Epstein L., Schneider M. (2003), "Recursive Multiple Priors", Journal of Economic Theory 113, 1-31.

[5] Epstein L., Wang T. (1994), "Intertemporal asset pricing under Knightian uncertainty", Econometrica, 62, 283-322.

[6] Ganderton P., Brookshire D. et alii (2000), "Buying insurance for disaster-type risk: experimental evidence", Journal of Risk and Uncertainty, 20, 3, 271-289.

[7] Hirshleifer D., Shumway T., (2003), "Good Day Sunshine: Stock Returns and the Weather", Journal of Finance, 1009-1032.

[8] Kahneman P., Tversky A., (1979), "Prospect Theory : an Analysis of Decision under Risk", Econometrica, 47, 263-291.

[9] Karni E. (1985) "Decision Making under Uncertainty: The case of statedependant preferences", Cambridge, Harvard University Press.

[10] Kreps D., Porteus E. (1978), "Temporal resolution of uncertainty and Dynamic choice theory", Econometrica, 46, 1, 185-200.

[11] Kunreuther H. (1996), "Mitigation disaster losses through insurance", Journal of risk and uncertainty, 12, 171-187.

[12] Machina M. (1989), "Dynamic Consistency and Non-Expected Utility Models of Choice under Uncertainty", Journal of Economic Litterature 27, 1622-1668. 
[13] McClelland G., Schulze W., Coursey D., (1993), "Insurance for lowprobability hazards: a bimodal response to unlikely events", Journal of risk and uncertainty, 7, 95-116.

[14] McClennen E. (1990), Rationality and Dynamic Choice, Cambridge University Press, Cambridge.

[15] Papon T. (2004), "L'influence de la durée d'engagement et du vécu dans les décisions d'assurance: deux études expérimentales", Cahiers de la $M S E \mathrm{n}^{\circ} 40$.

[16] Quiggin J., (1991), "Comparative Statics for Rank-Dependent Expected Utility Theory", Journal of Risk and Uncertainty, 4, 329-338.

[17] Slovic P., (2000), "The Perception of Risk", Earthscan Publications Ltd., London and Sterling.

[18] Tversky, A. and Kahneman, D. (1973), "Availability: a heuristic for judging frequency and probability", Cognitive Psychology, 5, 207-232.

[19] Tversky, A. and Kahneman, D. (1986), "Rational Choice and the Framing of Decisions", Journal of Business 59, 251-278.

[20] Yaari M., (1987), "The Dual Theory of Choice under Risk", Econometrica, 55, 95-105. 